\title{
Metacognición: mente y cerebro
}

\author{
Marianne Peronard Thierry* \\ Pontificia Universidad Católica de Valparaíso, Chile
}

\section{INTRODUCCIÓN}

Durante alrededor de 10 años, nuestro equipo se ha dedicado al estudio de la metacognición y su posible correlación con la comprensión y producción de textos escritos. En uno de los últimos trabajos resumíamos lo que había sido nuestro objetivo a lo largo de esas investigaciones, a saber, "comprobar si el conocimiento consciente de ciertos procesos cognitivos y de las características de los textos escritos contribuye a mejorar el desempeño de adolescentes en actividades relacionadas con el lenguaje escrito, es decir, a hacer de ellos comprendedores y productores de textos escritos más estratégicos" Peronard (2001).

\section{LA METACOGNICIÓN}

Este constructo, definido genéricamente por Flavell (1971) como "cognición de la cognición", ha sido tan discutible e interpretable como el

\footnotetext{
* Para correspondencia dirigirse a: Marianne Peronard (peronard@vtr.net), Instituto de Literatura y Ciencias del Lenguaje, Facultad de Filosofía y Educación, Pontificia Universidad Católica de Valparaíso, Casilla 4059, Avda. Brasil 2950, Valparaíso, Chile.
} 
concepto mismo de cognición. En términos no especializados, "cognición" significa "conocimiento (acción y efecto de conocer)" (DRAE 1992). Si bien esta definición parece ser muy pobre, al menos permite rescatar el hecho de que se trata de un proceso y de un producto. Por otra parte, el sufijo 'meta' no significa en este caso, 'junto a' o 'más allá de' si no que, al igual que lo hace Jackobson (1981) al hablar de función metalingüística, Flavell lo utiliza para referirse al hecho de que algo, un proceso, un conocimiento, se refleja sobre sí mismo, como la imagen en un espejo. Por ejemplo, el conocimiento de que algo se sabe o no se sabe, el conocimiento de las mejores estrategias para recordar, las estrategias para mantener la atención sobre algo en particular o para aprender algo de lo leído o escuchado.

Los primeros estudios, referidos a la memoria, buscaban descubrir cuánto sabían los niños acerca de la dificultad relativa que tendrían ellos para memorizar algo (proyectivo), cuándo creían haberlo memorizado (retrospectivo) y qué estrategias conocían para ayudarse a memorizar (introspectivo). Utilizando niños de diversas edades, llegaron a la conclusión de que los juicios de los sujetos acerca de la memoria a menudo eran errados pero que, con la edad, estos errores disminuían y los juicios se hacían más certeros, en otras palabras, que la introspección o expresión de la metacognición se hacía más certera.

El entusiasmo por los estudios metacognitivos pronto se hizo sentir, tanto entre los psicólogos como los psicolingüistas y especialmente entre los educadores, quienes vieron en ellos la posibilidad de facilitar el aprendizaje. La gran cantidad de trabajos diferentes que se realizaban trajo consigo, por una parte, una cierta confusión en cuanto al significado del término (Peronard 1999) y, simultáneamente, una mayor precisión en su alcance, gracias a la proposición de una serie de distinciones. La primera y tal vez la más nítida es aquella entre el autoconocimiento y el conocimiento de la mente de los otros, o el reconocimiento de que los otros tienen una mente parecida pero distinta a la nuestra. En Flavell (1971) esta distinción está poco elaborada, pero posteriormente se comienza a distinguir entre lo que se llama "teoría de la mente" y "metacognición", reservando este último término para el conocimiento de sus propios procesos y contenidos mentales. Una segunda distinción trajo considerables consecuencias metodológicas, aunque también aparece débilmente esbozada en esa obra, cuando distingue, por una parte, entre conocimiento de las capacidades de sí mismo en cuanto sujeto de la cognición, exigencias cognitivas de la tarea y las estrategias que conviene aplicar de acuerdo con los objetivos perseguidos con el proceso cognitivo y, por otra parte, lo que denomina experiencia metacognitiva, la sensación que tiene de lo percibido, que correspondería a lo que los filósofos denominan los qualia. Posteriormente, Flavell hace una nueva distinción -la que ha sido 
relacionada con el grado de conciencia del conocimiento metacognitivo- entre conocimiento declarativo (saber qué), más consciente y conocimiento procedural (saber hacer) más automatizado. Así, por ejemplo, el sujeto sabe que está prestando atención a un cierto objeto pero no sabe cómo lo hace su cerebro; sabe que conoce una determinada palabra, pero no sabe cómo lo hace para evocarla o decirla. Una distinción bastante similar, propuesta por Baker y Brown (1984) y acogida por otros estudiosos, es la que se plantea entre "conocimiento" y "control o regulación de la actividad".

Por otra parte, muchos investigadores comienzan a aplicar el concepto de metacognición a otros procesos cognitivos como la atención, la comunicación, la comprensión y la producción lingüística. Surgen así términos como metatención, metacomunicación, metacomprensión, metaproducción e, incluso, metalingüística para referirse a la conciencia del uso del lenguaje y no como originalmente en Jackobson (1981) relacionado con las funciones del lenguaje.

Como se señaló anteriormente, esta multiplicidad de estudios en áreas diferentes lleva a una cierta confusión y vaguedad terminológica, especialmente al cuestionarse el papel de la "conciencia" en la metacognición y, específicamente en el conocimiento y uso de estrategias (Peronard 1999). La mayoría de los estudiosos consideran que el conocimiento metacognitivo es concientizable, pero surgen dudas cuando se trata, por ejemplo, de la selección de estrategias para resolver problemas de comprensión o producción (Narens, Graf y Nelson, 1996). Una proposición muy oportuna es la de Karmiloff-Smith (1992) quien intenta compatibilizar la posición evolutiva de Piaget (1961) con la posición innatista de Fodor (1986) logrando así que la discusión, en muchos casos, no se continúe en términos polares -consciente, inconsciente- y comience a concebirse como un continuum dinámico que varía con la edad. A esta conclusión se llega gracias a los estudios desde la perspectiva evolutiva, sobre todo con escolares de diversas edades.

En todo caso, para que Flavell pudiera llegar a interesarse por un fenómeno como la metacognición fue necesario que la psicología recorriera un largo camino desde su nacimiento. La filosofía, en cambio, parece haber considerado que la cognición sí era un tema de discusión desde antiguo. En efecto, la discusión acerca de la posibilidad de que el ser humano pueda tener conciencia de los contenidos de su propia mente es de larga data en dicho ámbito. Ya Aristóteles disentía de la doctrina de las "ideas" de su maestro Platón como realidades "suprasensibles" separables de los seres individuales y con una existencia más allá de ellos. Consideraba, en cambio, que la cuidadosa observación de las formas permitiría llegar a lo universal en lo particular. Estos universales serían conceptos formados mediante procesos de abstracción sobre la base de repetidas sensaciones 
que llegan a la memoria, permitiéndonos, de este modo, experimentar lo mismo en lo individual y lo diverso. El hecho de que este filósofo haya sido el iniciador de dos disciplinas: la lógica o analítica preservada en lo que hoy se conoce como el Órganon y la psicología, representada por De Anima y una colección de artículos acerca de las sensaciones, la memoria, los sueños y el dormir reunidos en Parva Naturalia, permite concluir que el estagirita pensaba que los procesos mentales son tan observables como los hechos exteriores, es decir, que el hombre es capaz de un pensamiento reflexivo o introspección.

En el siglo III d.C., posiblemente a partir de las propuestas del neoplatónico Plotinio, se generaliza el interés por los estudios de la conciencia como base de la relación entre el mundo sensible y el mundo inteligible. Durante toda la Edad Media, el acceso a la conciencia o alma no es cuestionada, en especial por los pensadores cristianos, y tanto Tomás de Aquino como San Agustín apoyan y basan sus enseñanzas en creencias obtenidas mediante la introspección.

En el siglo XVII, Descartes (1954), partiendo del método inductivo y utilizando la introspección, llega a la conclusión de que la única verdad comparable con las verdades matemáticas de las que se puede tener plena certeza es el hecho de que estoy pensando (cogito ergo sum). Esta conclusión resultaba inevitable: si todo ha de ser puesto en duda es necesario que el individuo pensante no dude de su propia existencia, al menos en el momento en que está dudando. Esta existencia sería captada por el sujeto pensante en forma directa sin una idea intermediaria que lo represente a él mismo. El conocimiento que se pueda lograr de la existencia del resto de las cosas se obtiene mediante procesos inferenciales a partir de las correspondientes ideas presentes en forma innata en el intelecto (a priori). En consecuencia, los objetos inmediatos de los que el pensante tiene conciencia son siempre ideas. Al tomar conciencia de las ideas que forman el continuo flujo de nuestro pensamiento, es posible comprobar que algunas son entes de variadas características que se mueven, cambian e interactúan, y puesto que su existencia no está implicada en las ideas, ésta debe depender de la existencia de algo independiente de ellas: Dios. Esta distinción entre ideas (sustancia pensante) y entes materiales (sustancia extensa) conocida como "dualismo cartesiano" dio origen a innumerables discusiones y tomas de posición. Así, por ejemplo, algunos como Spinoza consideran que existe solo una sustancia que, en el hombre, se manifiesta en dos modalidades: una pensante y una extensa. Otros, en cambio, como el inglés Hobbes (1651), también en rechazo del dualismo cartesiano, se inclinan por una posición materialista, mientras que Locke (1690) toma una posición claramente dualista aunque niega todo tipo de innatismo. Para muchos, una debilidad 
fundamental de la posición cartesiana fue su imposibilidad de explicar a satisfacción de sus colegas cómo interactúan ambas sustancias. A ello se agrega su recurso a la existencia de Dios de quien dependerían, en última instancia, las ideas; este supuesto resultaba inaceptable para la mayoría de los filósofos posteriores.

En el siglo XIX, la psicología nace como disciplina independiente con la pretensión de seguir el modelo de las ciencias físicas. Como es bien sabido, los estudios llevados a cabo por psicólogos seguidores de la filosofía dualista como Wundt y Titchener dieron origen a la psicología experimental. Desde su perspectiva, los contenidos de la conciencia son similares a los de la experiencia inmediata y, por ello, irrefutables. Este supuesto fue motivo de serias disputas especialmente con filósofos como Brentano (1944), seguidor de Aristóteles, más inclinado al realismo que al idealismo y, sin embargo, firme sostenedor de la existencia de un alma personal. Este influyente profesor alemán definió la psicología como la ciencia del alma, considerando que su objeto debían ser los actos y procesos psíquicos y no los estados psíquicos. Sostenía que la introspección es falible, pues su captación no es inmediata, sino que es un proceso de observación inferencial y, por tanto, sujeto a error. A ello contribuiría, además, el hecho de que la conciencia está constituida no solo por un contenido sensorial (percepción y juicio o creencia), sino además por la valoración de dichos contenidos.

Estas prolongadas y no resueltas disputas, tanto entre materialistas y dualistas, así como entre estos últimos y, sobre todo, el advenimiento y auge del conductismo, terminaron por desacreditar la introspección como método para los estudios psicológicos. Junto con ello desaparece también el concepto de mente y la conciencia del horizonte del método científico. Surge y se generaliza el positivismo científico, que en psicología se realiza como "conductismo" cuya denominación proviene del principio básico que guía estas investigaciones: solo se puede estudiar científicamente la conducta, lo único perceptible, producto de algún tipo de cognición que estaría más allá de toda posibilidad de estudio.

Debieron pasar varias décadas de un período de ciencia normal bajo la égida del conductismo positivista antes de que se produjera un cambio de paradigma epistemológico que permitiera abrir la mente, "la caja negra", al estudio científico.

De este modo, el vacío epistemológico producto del debilitamiento del positivismo epistemológico vino a ser llenado por el cognitivismo. Uno de los primeros campos abordados desde esta perspectiva fue el de la ontogenia, es decir, el desarrollo de la inteligencia en el ser humano. En realidad, incluso antes y al margen de que esta revolución kuhniana afectara a la psicología ya se habían desarrollado corrientes psicológicas no conductistas. De hecho, uno 
de los pioneros en este campo, fue Freud (1900) el creador del psicoanálisis y Jean Piaget (1946) quien usó el método de observación clínica, que hoy denominaríamos "estudio de casos", desafiando la teoría de la probabilidad y el reinado de las estadísticas en boga en el siglo XX (Hacking (1995). Anteriormente, pero sin que llegara a conocimiento de los estudiosos occidentales, Vygotsky (1964) había hecho propuestas muy interesantes sobre el mismo tema que continúan en vigencia en la actualidad y que podríamos denominar una perspectiva sociopsicológica (Braaten 1991).

Con el surgimiento de la psicología cognitiva y, en cierta forma, el impacto de Freud y su psicoanálisis, los estudiosos empezaron a considerar como posible objeto de investigación el mundo interior del hombre Solso (1998).

Por otra parte, y lo que resulta fundamental para nuestras propias investigaciones, los psicólogos se sintieron muy interesados en el lenguaje, en parte gracias a que Chomsky (1965) pone en el centro de la arquitectura del lenguaje la sintaxis como un aspecto genéticamente determinado y la oración como unidad fundamental de la lengua. Logra así debilitar la supremacía del conductismo por su incapacidad para explicar la adquisición de este aspecto del lenguaje.

\section{NACE LA PSICOLINGÜÍSTICA}

Este interés dio origen al nacimiento a una interdisciplina denominada psicolingüística, cuyo punto de partida oficial fue un seminario organizado por el comité de Lingüística y Psicología del Social Science Research Council que reunió a lingüistas estructuralistas, teóricos de la información y psicólogos conductistas, todos bajo la égida del positivismo, seminario que dio origen a una publicación, denominada Psicolingüística, editado por Osgood, Sebeok y Diebold, obra publicada en 1974 y que debería ser realmente programática para esta nueva disciplina. Sin embargo, la psicología conductista había tenido que aceptar sus propias limitaciones producto de considerar que los resultados de los experimentos hechos con animales (perros, palomas, ratas) podían hacerse extensivos al ser humano, hecho que "repugnaba" a muchos psicólogos. Ambos hechos, la decadencia del conductismo y el impacto entre los científicos de esa época que tuvieron las proposiciones chomskianas, hicieron que la psicolingüística no prosperara en las líneas planteadas en el seminario y en su posterior publicación, sino 
que, por el contrario y coincidiendo con los cambios epistemológicos que afectaron tanto a la lingüística como a la psicología y, de hecho, a todas las ciencias humanas, la psicolingüística acogió el paradigma cognitivo.

El primer objetivo que surgió como natural y obvio -en gran medida impulsado por la búsqueda de la adecuación explicativa propuesta por Chomsky (1965) - fue el estudio de la adquisición de la lengua materna, hasta tal punto que el término "psicolingüística" llegó a ser sinónimo de los estudios en torno a la adquisición de la lengua materna. Posteriormente, y gracias al creciente interés de los psicólogos por el lenguaje y de los lingüistas por los procesos cognitivos subyacentes, los estudios se dirigieron hacia la comprensión y producción, tanto del lenguaje oral como al escrito.

Para enfrentar la complejidad de este objeto, cuyo estudio hasta entonces habían querido evitar los lingüistas, Saussure (1964), originalmente publicado en 1916 y Bloomfield (1933), la psicología cognitiva recurrió a metáforas más o menos iluminadoras que fueron aplicadas por los psicolingüistas. $\mathrm{La}$ primera fue la analogía con la computadora, que permitió que estos procesos fueran concebidos como productos del procesamiento de la información y la memoria como almacenes similares a los de estas máquinas: la memoria (operativa o de corto plazo) y la memoria de largo plazo. Este modelo es posteriormente rechazado y/o complementado con el modelo de las redes neuronales que trata de explicar el lenguaje en términos de una serie de asociaciones neuronales activadoras o inhibidoras de variados peso. Esta nueva perspectiva es conocida como "conexionismo" y si bien estas posiciones no han alcanzado consenso al interior de la comunidad científica, se han hecho esfuerzos por llegar a compatibilizarlos (Kintsch 1998), y han permitido avances interesante en la construcción de modelos que sustentan investigaciones novedosas.

En forma paralela, aunque sin enfatizar la relación con el lenguaje, los psicólogos de la escuela iniciada por Piaget (1946) continuaron el desarrollo de su disciplina. Cuando el psicólogo John Flavel -discípulo de Piaget y divulgador de sus ideas- comienza a trabajar con Wellman (Flavell y Wellman 1977), la psicología cognitiva estaba en pleno desarrollo y no dudan en usar con sus sujetos la introspección, tanto retrospectiva como proyectiva. Por eso mismo es que utilizan el término metacognición para designar el objeto de su investigación.

Ahora bien, en el siglo XX los avances tecnológicos permiten un nuevo enfoque para los estudios de la conciencia: el funcionamiento del cerebro. Nacen así las neurociencias (neuropsicología, neuropsicoanálisis, neurobiología y neurolingüística). La década de los 80 es testigo de una verdadera eclosión de estos estudios, cuyas publicaciones -a nivel de difusión y no de especialistas- empiezan a aparecer en la década del 90. Ya a comienzos de 
siglo se habían comenzado a desarrollar algunos estudios neurobiológicos motivados, en gran parte, por el famoso y conocido caso de Phineas Gage, trabajador ferrocarilero que sufrió un daño en la parte anterior del cerebro debido a una varilla que, al salir disparada, le atravesó la cabeza, entrando por la barbilla y saliendo por la parte superior del cráneo. Esto ocurrió en la década de $1840 \mathrm{y}$, si bien se recuperó físicamente, su personalidad cambió notablemente. Pero el hecho no logró publicidad, no trascendió al público general hasta pasado más de un siglo (Damasio et al. 1994).

Los primeros temas de los neuropsicólogos fueron ciertas psicopatologías específicas como trastornos de la conducta, del sueño, de la memoria y de la atención. Por su parte, el interés de los neurolingüistas se centró inicialmente solo en la relación entre el cerebro y las palabras, pero la idea del innatismo de la sintaxis propuesto por Chomsky invita a estos científicos a un enfoque más amplio para explicar cómo la evolución puede haber llegado, a partir de los cerebros de los monos - capaces solo de emitir gritos-a cerebros capaces de usar un lenguaje altamente estructurado y, al mismo tiempo, creativo (Calvin y Bickerton 2000). No son estos los únicos representantes de las neurociencias interesados en una perspectiva evolucionista del cerebro desde una posición claramente darwiniana; también la comparte el Dr. Vilayanur Ramachandran, uno de los más destacados neurocientíficos y prolífero autor de obras acerca del cerebro, desde la década de 1980: "I will suggest that many of these symptoms and disorders seem less bizarre when viewed from an evolutionary stanpoint, that is, from a Darwinian perpective. I also propose to give this discipline a new name-evolutionary neuro-psychiatry" (Ramachandran 2003: 98-9). Esta neuropsiquiatría evolutiva sería el resultado de aunar las perspectivas evolucionista de Darwin, las de Freud y de la neurología con instrumentos como la resonancia magnética y el PET de la sigla en inglés de "tomografía de emisión de positrones" procedimiento que, mediante la inyección de radioisótopos radioactivos, permite descubrir la mayor o menor actividad cerebral, midiendo las variaciones de la circulación sanguínea.

Sus investigaciones las realiza gracias a su rica experiencia con personas que manifiestan las más diversas patologías, producto, la mayoría de las veces, de accidentes (Ramachandran y Blackeslee 1998). Su trabajo lo llevó a distinguir dos tipos de desorden: uno en relación con las sensaciones subjetivas (qualia) asociadas a la percepción del mundo y otro asociado a la conciencia del yo. Los primeros problemas se manifiestan en sujetos que ven lo que no existe o que no ven lo que existe (para el investigador). Se pregunta cómo es posible que la actividad neuronal en el cerebro dé origen a los qualia, es decir, a la mente. El segundo problema es el de la conciencia del Yo, cuyas características serían la sensación de continuidad, de unidad 
o coherencia de sí mismo, de corporeidad, de ser agente de sus propias acciones y la capacidad de autorreflexión. Cada una de estas características o aspectos pueden verse alterados cuando el sujeto sufre diversas enfermedades cerebrales. Para explicar la mente y la conciencia del yo, Ramachandran (2003) sugiere que la evolución ha creado una estructura superior, una especie de ejecutivo central, que puede representar simbólicamente las representaciones sensoriales, una especie de metarrepresentación, que nos permitiría usar el lenguaje, tener conciencia del yo y anticipar las consecuencias de nuestras acciones, es decir, hipotetizar. Otros neurocientistas, interesados fundamentalmente en el mundo interior del ser humano, la conciencia y la identidad personal, han tomado una postura más bien freudiana, con la intención de fundar una subdisciplina que denominan "neuropsicoanálisis" que no utiliza tanto el estudio de los trastornos neurológicos, sino más bien los relatos de los pacientes de sus emociones, motivaciones, memoria, fantasía, sueños, alucinaciones y conciencia, temas, típicos del psicoanálisis. Anteriormente, se pensaba que este mundo interior no era susceptible de ser estudiado científicamente. "Sin embargo, en los últimos años -después del desprestigio del conductismo, la llegada de la tecnología de imagenología funcional del cerebro y la aparición de la neurobiología molecular- estos temas de repente han surgido de las sombras y se convirtieron en el centro de interés de muchos laboratorios neurocientíficos importantes alrededor del mundo" (Solms, M. y Turnbull, O. 2004: xiii). El lenguaje pasa así a ser fundamental como herramienta para llegar a la conciencia y la introspección una forma validada para llegar a la conciencia. Pero el hecho de que un psiquiatra pueda llegar a la conciencia de otros, interpretando lo que el paciente dice no presupone que el paciente o el psiquiatra puedan llegar a su propia conciencia. La relación entre conciencia y lenguaje no es tan directa y unívoca en estos casos. Primero, porque, si bien parece ser irrefutable que el paciente debe tener conciencia de lo que está pensando para poder enunciarlo verbalmente, lo que piensa no corresponde necesariamente a lo percibido en su oportunidad. Bien se sabe que cuando se evocan episodios guardados en la memoria de largo plazo, éstos se alteran cada vez que se traen a la conciencia. Los cambios se introducen involuntariamente y se debe a que la persona que vivió los episodios no es exactamente igual a la que los reproduce. En segundo lugar, porque el psiquiatra debe interpretar lo dicho por el paciente y en ello no pueden dejar de influir sus preconcepciones, tanto científicas como acerca del paciente. Por último, la información no dice relación, en absoluto, con las estrategias cognitivas que el paciente pone en juego para llevar a la conciencia dicha información. Ni siquiera arroja luz acerca de cómo funciona el cerebro para que el sujeto pueda hablar y decir lo que quiere decir: "Try to introspect the next time 
you talk. You will hear well formed sentences coming tumbling out if your mouth, but without any knowledge of what entity formed them with the appropiate syntax" (Koch 2004: 206).

Indudablemente que las neurociencias han permitido descubrir mucho acerca del cerebro, pero falta aún mucho por descubrir. Como se señaló anteriormente, la idea de que una parte del cerebro, específicamente de la corteza cerebral, puede interpretar las actividades neuronales de estructuras inferiores, se parece mucho a la idea del homúnculo: "In fact, what I am calling a metarepresentation bears un uncanny resemblance to the homunculus that philosophers so much delight in debunking" (Ramachandran 2003: 116).

Las neurociencias han aumentado enormemente el conocimiento que se tiene acerca de cómo funciona nuestro cerebro, y su avance resulta fascinante. Sin embargo, casi al final de uno de sus libros, Ramachandran señala que: "The problem of mind and matter, substance and spirit, illusion and reality, has been a major preoccupation of both Eastern and Western philosophy for millennia, But very little of lasting value has emerged" (Ramachandran y Blakeslee 1998: 228).

Ahora bien, el hecho de que los neurólogos no hayan podido llegar a un consenso ni descubrir con certeza qué parte de nuestro cerebro entra en juego para tener sensaciones subjetivas ni conciencia del Yo, no tiene gran consecuencia para el concepto de metacognición. Por el contrario, estos estudios refuerzan la existencia de la capacidad de autorreflexión y, de hecho, es uno de los fenómenos en los que basan sus investigaciones. Por otra parte, también se refuerza la idea de la existencia de lo que denominan "zombies" en la mente, que se manifiestan en las actividades inconscientes, automatizadas, probablemente producto de la experiencia.

Estos numerosos y variados estudios sugieren que, dada la importancia de la metacognición para la educación y, en especial, para la enseñanza del lenguaje, la metacognición debería seguir estudiándose desde el punto de vista psicológico, incorporando algunas de las aportaciones de la neurología. Por ejemplo, es importante reconocer que es posible que las estrategias utilizadas durante la lectura o la escritura sean conscientes y, por tanto, enseñables teóricamente o ya estén automatizadas gracias a que se han aplicado conscientemente con frecuencia, es decir, se haya desarrollado la habilidad. Por último, es posible que sean producto de los zombies de los que hablan los neurólogos, y no del aprendizaje.

Es cierto que la distinción entre actividades conscientes, por un lado, y actividades automatizadas, por otro, ya había sido insinuada por los estudiosos de la metacognición, aunque, obviamente, sin poder aportar pruebas empíricas como lo han hecho los neurólogos. Esta concepción se observa 
en la distinción, mayoritariamente aceptada, entre conocimiento declarativo y conocimiento procedural, es decir, entre saber qué hago y saber cómo lo hago. Un conocimiento típicamente procedural es, por ejemplo, andar en bicicleta: sé que estoy montado en ella y que voy avanzando pero no sé qué órdenes mi cerebro pueda haberle dado a mis músculos para que se muevan como deben hacerlo. Ni el más eximio de los ciclistas sabría cómo explicárselo a quien quiera aprender.

Retomando el problema de la metacognición y la mente, por lo dicho hasta ahora, este se podría resumir como sigue: las estrategias son conscientes e intencionadas, y en consecuencia, sujetas a la autorreflexión y al control. El conocimiento procedural no es consciente $y$, consecuentemente no podría ser sujeto de la metacognición, ni enseñable. Esta conclusión parece ser exageradamente extrema. Desde una perspectiva ontogenética, el problema no parece que pudiera plantearse tan taxativamente; de hecho, es evidente que parte del conocimiento procedural es inicialmente consciente y solo posteriormente, a medida que ejercita y se automatiza pasando a ser realizada en forma automática. Para confirmarlo, basta con recurrir a nuestras propias experiencias con la lectura y la escritura -por limitarnos a fenómenos psicolingüísticos- $\mathrm{o}$ al manejo de una bicicleta o automóvil y tantas otras actividades que ya adultos realizamos automáticamente.

Resulta curioso que, a pesar de todos los estudios realizados en torno a la mente y al cerebro, en relación con el lenguaje aún no haya sido posible encontrar una explicación para la adquisición de la sintaxis de nuestra lengua materna. Esta habilidad la adquirimos sin tener conciencia de ello ni del esfuerzo que significa, para luego hacer uso de ese conocimiento automática pero, al mismo tiempo, creativamente. 


\section{REFERENCIAS BIBLIOGRÁFICAS}

BACKer, Linda y AnN Brown. 1984. Cognitive monitoring in reading. En James Flood (ed.), Inderstanding Reading Comprehention: Cognition, Language and the Structure of Prose. Newark, Delaware: I.R.A, pp. 21-43.

Bloomfield, Leonard. 1933. Language. New York: Holt, Reinhard \& Winston.

BraAten, IVor. 1991. Vygotsky as precursor to metacognitive theory: The concept of metacognition and its roots. Scandinavian Journal of Educational Research 35, 3: 179192.

Brentano, Klemen. 1944. Psychologie du point de vue empirique. Paris: Aubier.

Calvin, William H. y Derek Bickerton. 2000. Lingua ex Machine. Cambridge, Mass.: MIT Press.

Сномsкy, Noam. 1965. Aspects of the theory of Syntax. Cambridge, Mass: MIT Press.

Damasio, Hanna, Thomas Grabowsky, Randa Frank, Albert Galaburda y Antonio Damasio. 1994. The return of Phineas Gage: The skull of a phamous patient yields clues about the brain. Science 264: 1102-1105.

Descartes, Rene. 1954. La crítica del método. Rio Piedras: Editorial de la Universidad de Rio Piedras.

Flavell, John. 1971. First discussant's comments: what is memory development the development of? Human Development 14: 272-278.

Flavell, John y Wellman. 1997. Metamemory. En R.V. Kail y J. W.Hagen (eds.), Perspectives on the Development of Memory and Cognition. Hillsdale: N.J. Erlbaum.

Fodor, Jerry. 1986 (1983). La Modularidad de la Mente, Madrid: Ediciones Morata.

HACKING, IAN. 1995. Comments on scientific realism about some chemical entities. Foundations of Science, Vol. No 4: 537-542.

Jakobson, Roman. 1981 (1963). Lingüistica y Poética. Madrid: Cátedra.

Karmilov-Smith, Annette. 1992. Beyon Modulariry: a Developmental Perspective. Cambridge, Mass: M.I.T. Press.

KintsCh, Walter. 2004 (1998). The Construction-Integration model of text comprehension and its implications for instruction. In R. Ruddell y N. Unrau (eds.), Theroretiocal Models and Processes of Reading. 5th Edition, International Reading Association.

Koch, Christof. 2004. The Quest of Consciousness. U.S.A: Roberts \& Co.

Osgood, Theodor, Thomas A. Sebeok y Diebold. 1974. Psycholinguistics. Barcelona: Planeta. 
Peronard, Marianne. 1999. Metacognición y Conciencia. En G.Parodi (ed.), Discurso congnición y Educación. Ensayos en honor a Luis A. Gómez Macker. Valparaíso: Ediciones Universitarias, pp.43-57.

2001. El conocimiento metacomprensivo en escolares chilenos de educación básica. Signos XXXIV, ํ 49.50: 149-164.

Piaget, Jean. 1961 (1946). Desarrollo del símbolo en el niño: México: Fondo de Cultura Económica.

Ramachandran, Vilayanur y Sandra Blakeslee. 1998. Phantoms in the Brain. Nueva York: Harper Collins.

Ramachandran, Vilayanur. 2003. The Emerging Mind. London: Profile Books, Ltd.

2004. A Brief Tour of Human Consciousness. New York: Pearson Education, Inc.

Saussure, Ferdinand. 1964 (1916). Curso de Lingüistica General. Buenos Aires: Losada S.A.

Solms, Mark y Oliver Turnbull. 2004 (2002). El Cerebro y el Mundo Interior. México: Fondo de Cultura Económica.

Solso, Robert L. 1998. Cognitive Psychology. Boston: Allyn y Bacon.

Narrens, Louis, Aurora Graf y Thomas O. Nelson. 1996. Metacognitive Aspects of Implicit/ Explicit Memory. En L. M. Reder (ed.), Implicit Memory and Metacognition, Mahwah, N.J.: Lawrence Erlbaum, pp. 137-170.

Vygotsky, Leopold. 1964. Pensamiento y Lenguaje Buenos Aires: Lautaro. 